\title{
Pathological changes in the terminal parts of Purkyně cell axons in the dentate nucleus of the cerebellum ${ }^{1}$
}

\author{
IVAN JANOTA \\ From the Department of Neuropathology, Institute of Psychiatry, and the \\ Maudsley Hospital, Denmark Hill, London
}

SUMMARY This paper describes abnormal argyrophilic swellings and rings in the dentate nucleus of the cerebellum in 46 cases of various human pathological conditions. The swellings represent accumulations of neurofilaments in the terminal parts of Purkyně cell axons. They may be evidence of disturbed functions of the Purkyně cell terminals due to different causes and common to many pathological conditions. These are as varied as acute ischaemic cerebellar cortical lesions, liver failure, and chronic nervous system degenerations, such as progressive supranuclear palsy and Friedreich's ataxia, and include transmissible ones like Creutzfeldt-Jakob disease.

In ischaemic cerebellar cortical disease, liver failure, and various degenerations of the nervous system (Table 1), prominent rounded rings and swellings, which are not conspicuously present in normal controls, appear on the terminal parts of Purkyně cell axons in the dentate nucleus of the cerebellum in sections impregnated with neurofibrillary silver methods. On electron microscopy the swellings contain accumulations of $10 \mathrm{~nm}$ neurofilaments. This change is similar to the transient appearance of argyrophilic boutons which occurs in various parts of the central nervous system when axons are cut or cell bodies destroyed. It is known to occur in the terminal parts of Purkyně cell axons in the Deiters' nucleus after experimental lesions in the cat cerebellar cortex. The findings in ischaemic cerebellar disease where there is damage to the Purkyně cell bodies are similar to those in the cat experiment. In liver failure, where neither the Purkyně cell bodies nor the dentate nerve cells appear to be damaged, the reason for the change may be a metabolic disturbance. The change in progressive supranuclear palsy, where the rest of the Purkyně cell is normal but where there is a loss of dentate nerve cells, may be an example of a retrograde transneuronal process.

1 A preliminary communication on this subject was given at the Meeting of the British Neuropathological Society, London, February 1972.

\section{METHODS}

The cerebellum of some 300 cases including patientsi with cerebellar lesions and controls where there wasp no cerebellar disease was examined. The materia ${ }^{\mathcal{C}}-$ was surveyed for pathological changes in the dentate nucleus with special reference to the occurrence of

TABLE 1

PATHOLOGICAL CONDITIONS IN 46 CASES

\begin{tabular}{cr}
\hline Pathological conditions & Number of cases \\
\hline I. Ischaemic lesions in cerebellar cortex & 13 \\
Cardiac arrest at operation & 1 \\
Cardiac surgery & 7 \\
Carbon monoxide poisoning & 1 \\
Barbiturate poisoning & 1 \\
Drug overdose & 1 \\
Hypertension & 2 \\
II. Liver failure & 13 \\
Necrosis & 5 \\
Cirrhosis & 3 \\
Cirrhosis and portocaval anastomosis & 2 \\
Obstructive jaundice & 2 \\
Hepatoma & 1 \\
III. Degenerations of nervous system & 15 \\
Progressive supranuclear palsy & 6 \\
Friedreich's ataxa & 3 \\
Progressive cerebellar degeneration and & \\
myoclonus & 1 \\
Spinocerebellar degeneration & 1 \\
Creutzfeldt-Jacob disease & 1 \\
Alzheimer's disease & 1 \\
Cerebellar degeneration with carcinoma & 1 \\
Refsum's disease & 1 \\
IV. Other conditions & 5 \\
Leukaemia & 2 \\
Encephalitis & 2 \\
Acute tracheobronchitis & 1 \\
\hline
\end{tabular}


TABLE 2

PATHOLOGICAL CHANGES IN CEREBELLUM AND GENERAL DATA OF CASES

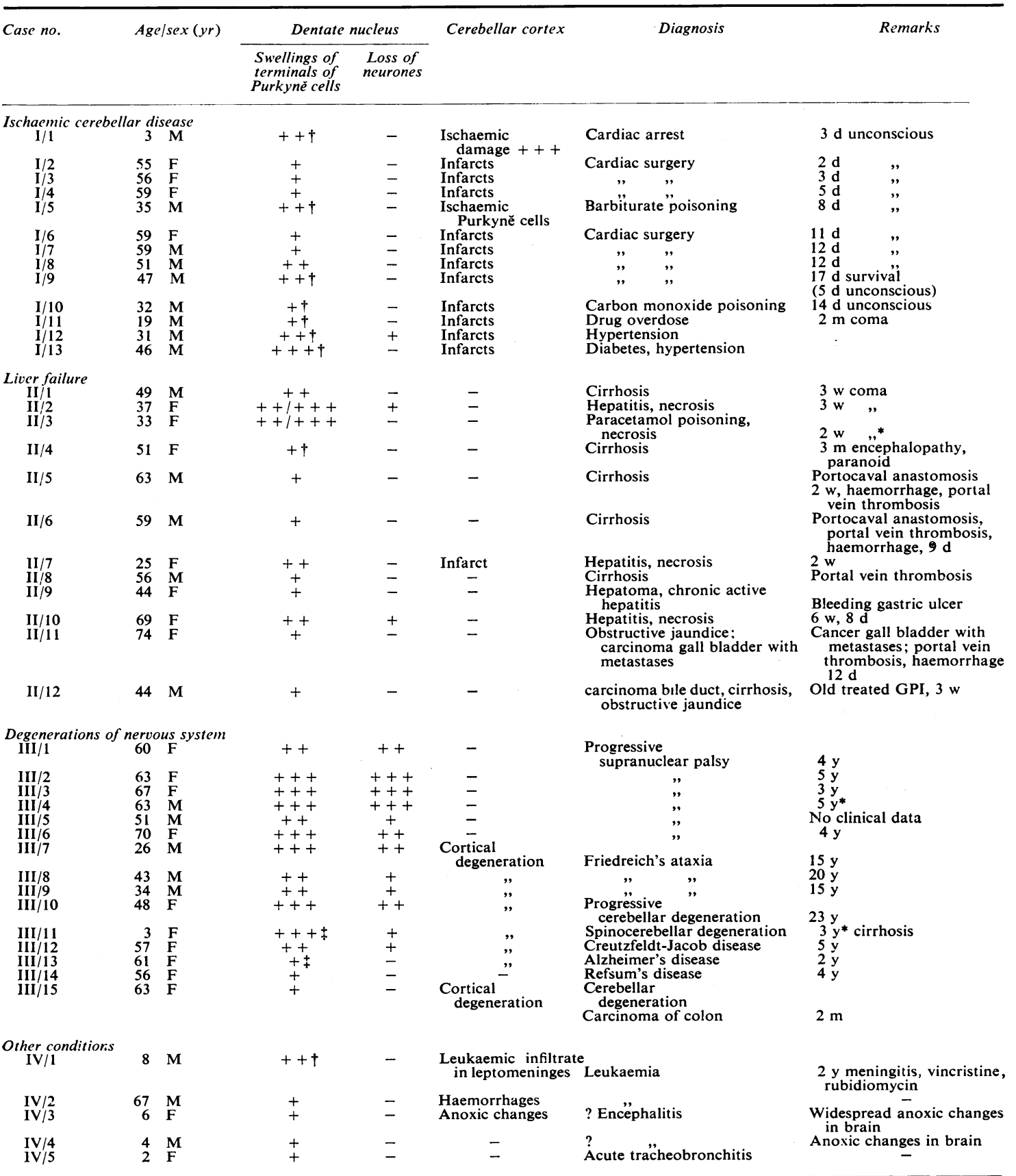

* Examined by electron microscope.

† localized.

₹redominantly dense swellings.

$+=$ degree of severity of changes.

- = absence or changes.

$\mathrm{y}=$ years. $\mathrm{m}=$ months. $\mathrm{w}=$ weeks. $\mathrm{d}=$ days. 
argyrophilic swellings around the bodies and processes of the dentate nerve cells and to the loss of the dentate nerve cells. In the normal controls it was unusual to encounter any argyrophilic swellings. Forty-six cases where there were definite pathological changes were selected for study (Table 1). The general data about the cases and the degree and severity of the pathological changes are given in Table 2. The pathological change was roughly assessed as mild $(+)$ to severe $(+++)$. One plus $(+)$ indicated the presence of 1 to 3 argyrophilic swellings in every high power microscope field, while three pluses $(+++)$ corresponded to 10 or more swellings in a high power field. The loss of dentate nerve cells was classified from mild $(+)$ to severe $(+++)$ where no nerve cells were seen in some high power fields.

Sections of the cerebellum including the dentate nucleus embedded in paraffin wax were impregnated with silver according to the following techniques: Palmgren, Glees, and Bodian. Frozen sections were treated according to von Braunmühl. A variety of conventional staining techniques was employed apart from silver impregnation. Small blocks of formalin fixed material from five cases and from a control were postfixed in osmic acid and processed for electron microscopy, and some blocks were impregnated with silver before processing for electron microscopy.

\section{PATHOLOGICAL FINDINGS}

Abnormalities in the dentate nucleus were found in cases with recent cerebellar cortical lesions, liver failure, degenerations of the nervous system, and some other conditions, and the findings in the main groups are presented below. The principal pathological features common to all the groups are briefly discussed first.

Light microscopy of sections impregnated with silver showed prominent rings and knobs 2 to $10 \mu$ in diameter, often in continuity with nerve fibres, around the bodies and processes of the nerve cells in the dentate nucleus (Figs 1-3). Only small fine argyrophilic rings were occasionally found in the controls. When there was a loss of nerve cells in the dentate nucleus the rings and knobs formed clusters in their place (Fig. 1). They appeared as granular clumps in sections stained with haematoxylin and eosin or periodic acid Schiff.

Electron microscopy revealed whorls or ring-like accumulations of bands of $10 \mathrm{~nm}$ neurofilaments (Figs 4 and 5). Apart from neurofilaments the swellings contained mitochondria. The swellings were often seen along the border of nerve cells (Fig. 4) or closely adjacent to nerve cell processes, and some were continuous with nerve fibres (Fig. 5). Since this was post-mortem material there was much loss of structural detail, especially of vesicles and of cell membranes, and only rarely could a synaptic thickening of cell membrane be identified. In sections impregnated with silver before processing for electron microscopy silver granules were concentrated amongst the neurofilaments in the swellings and in nerve cell processes.

The pathological findings in the main groups are shown in Tables 1 and 2.

ISCHAEMIC DISEASE OF CEREBELLUM (CASES I/1-13) This group included patients who underwent cardiac surgery and died after intervals of unconsciousness from two to 17 days (cases $I / 2-4$ and 6-9). There was no loss or any obvious damage to the nerve cells in the dentate nucleus in this group. The accumulations of argyrophilic swellings in the dentate nucleus in such cases roughly corresponded to areas of cerebellar cortical ischaemic change with damage to Purkyner cell bodies. In one patient, a 3 year old boy (case I/1) who survived unconscious for three days after cardiac arrest at an operation, there was a widespread irreversible ischaemic damage to the Purkyne cells and the argyrophilic change in the dentate nucleus was particularly extensive and severe (Fig. 2). Patients with old cerebellar cortical scars, usually associated $\stackrel{?}{\mathbb{P}}$ with cerebrovascular disease did not show any argyrophilic change, nor was the change found in patients surviving for less than two days. The change was not found when there were recent or old vascular lesions involving the dentate nucleus itself.

LIVER FAILURE (CASES II/1-13) Liver failure was due to various causes: necrosis, following hepatitis or paracetamol poisoning, cirrhosis aggravated by portal vein thrombosis, which in two instances followed portocaval anastomosis, obstructive jaundice, and hepatoma. Most cases had hepatic encephalopathy. The duration of the fatal phase of the disease was relatively short, up to three weeks, in some cases ending with haemorrhage in the alimentary tract. There were changes in astrocyte nuclei and Bergmann glia which are usual in liver disease. The Purkyně cells as well as the dentate nerve cells were generally well preserved and the pathological change was focused on the Purkyner cell terminals. The swellings were usually more prominent where there was liver necrosis. They were particularly numerous in a $\mathbf{3 7}$ year old woman with massive liver necrosis after hepatitis (case II/2) where there was also some loss of nerve cells in the dentate nucleus, in a 69 year old woman with liver necrosis after hepatitis (case II/10), and in case II/3, a 33 year old woman with acute liver necrosis due to paracetamol poisoning. The general clinical and pathological 


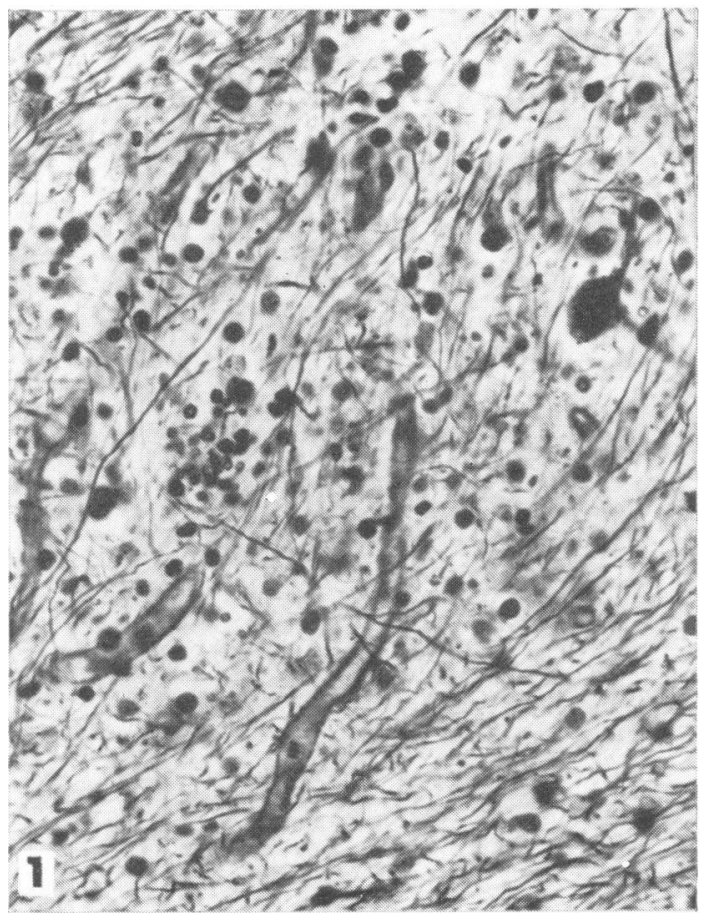

FIG. 1. Case III/4. Dentate nucleus with rings, clubs, and circles representing altered terminals of Purkynĕ cells. The cluster near the middle has remained in the place of a nerve cell. A surviving nerve cell is seen near the edge on the right. Progressive supranuclear palsy. Palmgren, $14 \mu, \times 200$.

FIG. 2. Case I/1. Altered enlarged terminals of Purkynè cells around a dentate nerve cell and its processes. Some of the ring and club-shaped swellings are continuous with slender nerve fibres. Two different focal planes of the same section. From a 3 year old child who died three days after cardiac arrest at operation. Palmgren, $14 \mu, \times 600$.

FIG. 3. Case III/10. Abnormal axonal terminals of Purkynè cells around two dentate neurones in two different focal planes of the same section. From a 3 year old girl with an atypical spinocerebellar degeneration. Bodian/Neutral Red, $20 \mu, \times 600$.
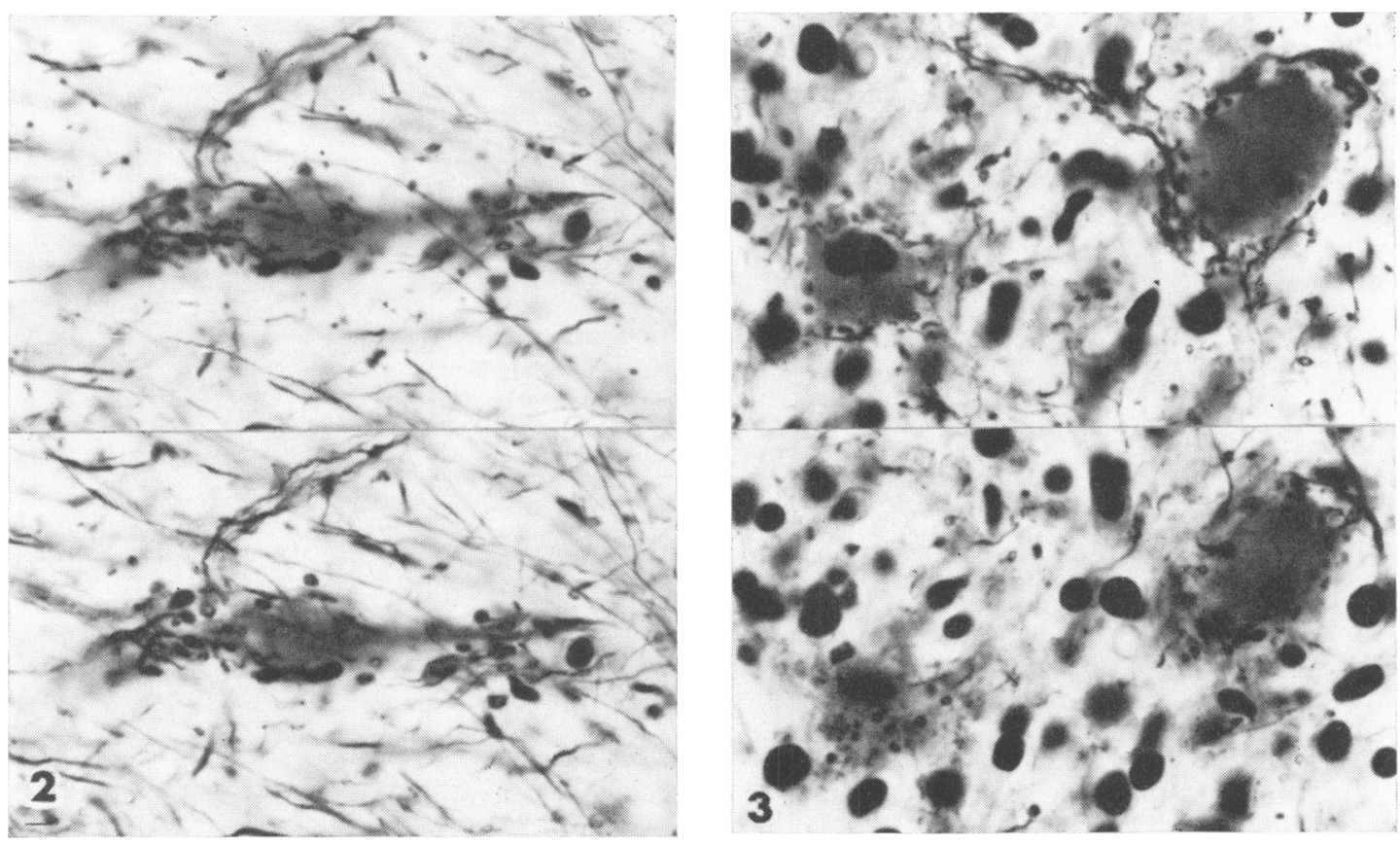


(1)

FIG. 4. Case III/4. Accumulations of neurofilaments in swollen axonal terminals in the dentate nucleus. The dense structure in the upper part of the picture is a process of a dentate nerve cell. Note the ring-like arrangement of neurofilaments in the bottom right hand corner. Progressive supranuclear palsy, $\times 7,500$.

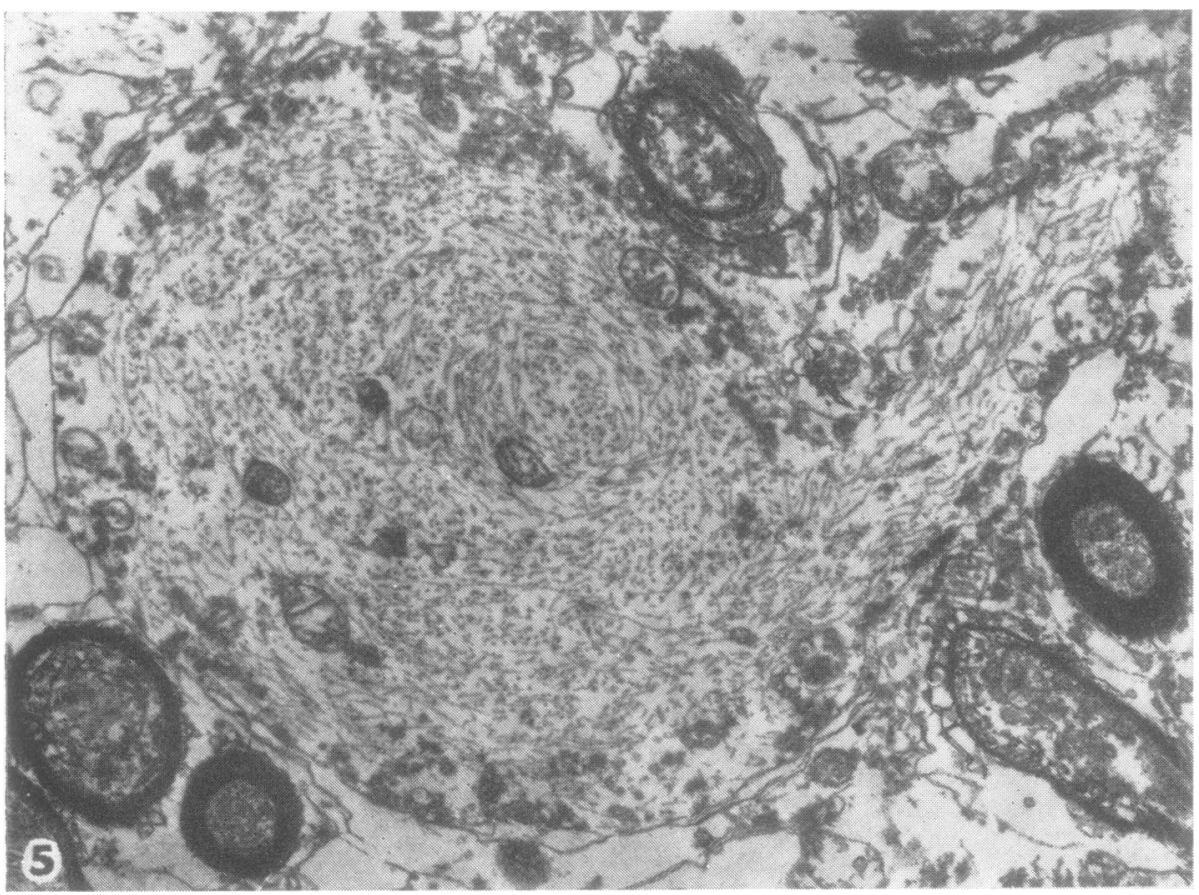

FIG. 5. Case III/4. A slender terminal part of an axon on the right continuous with a swelling composed of whorls of $10 \mathrm{~nm}$ neurofilaments. Progressive supranuclear palsy, $\times 15,000$. 
findings in this case were reported by Toghill, Williams, Stephens, and Carroll (1969). Many other cases of liver disease not included in this group showed only scanty argyrophilic swellings; they were usually cases of cirrhosis.

DEGENERATIONS OF NERVOUS SYSTEM (CASES III/1-15) In this group the clinical history, in contrast with the first two groups, was long, usually several years. In progressive supranuclear palsy (cases III/1-6) the findings were unique in that there was always severe loss of dentate nerve cells. The Purkyner cells in the cerebellar cortex were within normal limits. The argyrophilic swellings were widespread and they appeared to remain marking the place previously occupied by the dentate nerve cells (Figs 1, 4, and 5). The clinical and neuropathological details of cases III/1-4 were reported elsewhere (Behrman, Carroll, Janota, and Matthews, 1969). Case II/5 was a 51 year old man with dementia and with atrophy of the midbrain. The clinical information was limited but the histological findings were characteristic of progressive supranuclear palsy. Case II/6 was a 70 year old woman with a history of anarthria for four years and with akinetic mutism, again with histological changes of progressive supranuclear palsy. In the other conditions in the group of degenerations of the nervous system there were moderately severe accumulations of argyrophilic structures with some loss of nerve cells in the dentate nucleus as well as degenerative changes in the cerebellar cortex. They included Friedreich's ataxia (cases III/7-9), Creutzfeldt-Jakob disease, Alzheimer's disease, atypical spinocerebellar degeneration and liver cirrhosis in a 3 year old child (Fig. 3) (case III/10), cerebellar degeneration associated with carcinoma of the colon, and Refsum's disease. The clinical and biopsy findings in the case of Refsum's disease were reported by Rake and Saunders (1966). In case III/11, a 48 year old woman with 21 years' history of myoclonus and ataxia, there were particularly severe changes in the dentate nucleus as well as degeneration of the cerebellar cortex.

OTHER CONDITIONS (CASES IV/1-5) In several other cases, not included in the first three groups, the change was also prominent. They were an 8 year old boy (case IV/1) with leukaemic infiltration in the leptomeninges and around blood vessels, a 67 year old man (case IV/2) with leukaemia and with focal haemorrhages in the cerebellum, a 6 year old girl (case IV/3) with two days' history of vomiting and drowsiness and with an acute encephalitis and widespread anoxic changes in the nervous system, a 4 year old boy (case IV/4) also with acute encephalitis, and a 2 year old girl (case IV/5) who died suddenly of acute tracheobronchitis.

\section{DISCUSSION}

Argyrophilic swellings around the nerve cells in the dentate nucleus in man were described in a combined degeneration of the globus pallidus and dentate nucleus (Neumann, 1959) and in progressive supranuclear palsy (Behrman et al., 1969). Similar findings were reported in Creutzfeldt-Jacob disease (Beck, Daniel, Matthews, Stevens, Alpers, Asher, Gajdusek, and Gibbs, 1969).

The presence of argyrophilic neurofibrils in the nerve cells and their processes is well recognized in man and in animals and electron microscopy has shown that neurofilaments are present in the structures that are argyrophilic (Guillery, 1965; Wisniewski, Terry, and Hirano, 1970; Terry, 1971). Some axonal terminals or boutons are argyrophilic and contain neurofilaments (Ramón y Cajal, 1952-55; Gray and Guillery, 1966). Ramón y Cajal describes swellings on terminal branches of Purkyně cell axons in the 'cerebellar olive' in young animals, corresponding to the human dentate nucleus, and illustrates minute neurofibrillary rings on the collaterals of Purkyně cell axons in the cerebellar cortex of an adult dog. Such structures resemble the fine small argyrophilic rings rarely found in the dentate nucleus of the controls examined in the course of the present study. In certain situations the axonal terminals enlarge and the neurofilaments transiently accumulate in the course of experimental degeneration. Examples of this form of degeneration include the lateral geniculate body in cats four to five days after transection of the optic nerve (Szentágothai, Hámori, and Tömböl, 1966) and in monkeys after enucleation of the eye (Colonnier and Guillery, 1964), and the optic tectum of chicks eight days after section of the optic nerve (Gray and Hamlyn, 1962). Rogers (1972), who studied degenerating boutons in the spinal cord after spinal hemisection in the cat, observed that the neurofibrils accumulated in the preterminal axon and its continuation with the bouton and not just in the bouton alone.

Axonal terminals usually degenerate without becoming argyrophilic and without passing 
through a phase of accumulation of neurofilaments (Alksne, Blackstad, Walberg, and White, 1966) and caution is necessary in equating argyrophilia with the presence of neurofilaments in boutons. Thus Heimer (1967) observed that degenerating boutons in the olfactory cortex lacked filaments on electron microscopy and yet appeared argyrophilic in Glees sections, and Walberg (1964) made a similar observation in the inferior olive. Purkyně cell terminals, however, are among those which enlarge and where neurofilaments do accumulate in the course of degeneration-that is, in the lateral vestibular nucleus three to four days after lesions in the cerebellar vermis in cat (Walberg and Mugnaini, 1969).

The findings in the group of cerebellar ischaemic disease are consistent with the experimental observation that neurofilaments accumulate transiently in the endings of an irreversibly damaged or destroyed Purkyně cell. In human pathological material it is seldom possible to determine with any accuracy the time of onset of a pathological process, but the appearance of argyrophilic swellings two to 17 days after cardiac surgery is roughly in agreement with the interval when similar change occurs in animal experiments. The conditions in case $I / 1$, a child surviving unconscious for three days after cardiac arrest, were in particular reminiscent of those in Walberg and Mugnaini's (1969) experiment. In the other cases with cerebellar cortical vascular lesions it was impossible to prove that the change occurred in irreversibly damaged Purkyně cells, as seemed likely, or perhaps in cells that could have recovered.

In man the accumulation of neurofilaments does not occur only when the Purkyně cell is destroyed or its axon cut as in animal experiments. In liver failure, usually with encephalopathy and with a relatively short history of the terminal phase, the pathological abnormality was confined to Purkyně cell terminals. The bodies and axons of Purkyně cells were within normal limits on light microscopy and there was generally no abnormality of the nerve cells in the dentate nucleus. It seems possible that there occurs a transient metabolic disturbance in the axonal terminal in these cases. Weiss and Mayr (1971) in a discussion of axonal flow suggest that neurofilaments represent a 'momentary configuration of flux of molecular assembly and redispersion'; when the axoplasmic flow is disturbed - for instance, by constriction-eddies in the axoplasm are reflected in the appearance of interlacing neurofilaments. Variation of physical conditions also can influence the ease of staining of neurofilaments and boutons: it is possible to stain more boutons in the brain-stem of lizards when the temperature is lowered (Boycott, Gray, and Guillery, 1961). The clinical data in our material do not allow any speculation about the possible mechanism in liver disease.

The pathological changes in some of the degenerations of the nervous system are complex, with involvement of both the cerebellar cortex and the dentate nucleus, but they seem more akin to progressive supranuclear palsy than to the conditions in the other groups. In progressive supranuclear palsy it seems that dentate neurones disappear and that neurofilaments accumulate in the 'disconnected' terminals of Purkyně cell, and that the reaction goes on for a longer time without any obvious change in the rest of the Purkyně cell in spite of several years clinical history. This would be an example of retrograde transneuronal process.

I thank Dr. Sabina J. Strich for her interest and advice and Mr. A. J. Davey for assistance in preparing the illustrations. I am grateful to the many colleagues who sent brains for pathological examination. This work was supported by a grant from the Research Fund of the Bethlem Royal and Maudsley Hospitals.

\section{REFERENCES}

Alksne, J. F., Blackstad, Th. W., Walberg, F., and White. L. E., Jr. (1966). Electron microscopy of axon degeneration: a valuable tool in experimental neuroanatomy. Ergebnisse der Anatomie und Entwicklungsgeschichte, Band 39, Heft 1. Springer: Berlin.

Beck, E., Daniel, P. M., Matthews, W. B., Stevens, D. L., Alpers, M. P., Asher, D. M., Gajdusek, D. C., and Gibbs, C. J., Jr. (1969). Creutzfeldt-Jakob disease. The neuropathology of a transmission experiment. Brain, 92, 699-716. Behrman, S., Carroll, J. D., Hanota, I., and Matthews, W. B. (1969). Progressive supranuclear palsy. Clinico-pathological study of four cases. Brain, 92, 663-678.

Boycott, B. B., Gray, E. G., and Guillery, R. W. (1961). Synaptic structure and its alteration with environmental temperature: A study by light and electron microscopy of the central nervous system of lizards. Proceedings of the Royal Society. Series B, 154, 151-172.

Colonnier, M., and Guillery, R. W. (1964). Synaptic organization in the lateral geniculate nucleus of the monkey. Zeitchrift für Zellforschung und mikroskopische Anatomie, 62, 333-355. 
Gray, E. G., and Guillery, R. W. (1966). Synaptic morphology in the normal and degenerating nervous system. In International Review of Cytology, Vol. 19, pp. 111-182. Edited by G. H. Bourne and J. F. Danielli. Academic Press: New York.

Gray, E. G., and Hamlyn, L. H. (1962). Electron microscopy of experimental degeneration in the avian optic tectum. Journal of Anatomy, 96, 309-316.

Guillery, R. W. (1965) Some electron microscopical observations of degenerative changes in central nervous synapses. Progress in Brain Research, 14, 57-76.

Heimer, L. (1967). Silver impregnation of terminal degeneration in some forebrain fiber systems: a comparative evaluation of current methods. Brain Research, 5, 86-108.

Neumann, M. A. (1959). Combined degeneration of globus pallidus and dentate nucleus and their projections. Neurology (Minneapolis), 9, 430-438.

Rake, M., and Saunders, M. (1966). Refsum's disease: a disorder of lipid metabolism. Journal of Neurology, Neurosurgery, and Psychiatry, 29, 417-422.

Ramón y Cajal, S. (1952-55). Histologie du Système Nerveux de l'Homme et des Vertébrés. 2 vol. Consejo Superior de Investigaciones Científicas: Madrid.

Rogers, D. (1972). Ultrastructural identification of degenerating boutons of monosynaptic pathways to the lumbosacral segments in the cat after spinal hemisection. Experimental Brain Research, 14, 293-311.
Szentágothai, J., Hámori, J., and Tömböl, T. (1966). Degeneration and electron microscope analysis of the synaptic glomeruli in the lateral geniculate body. Experimental Brain Research, 2, 283-301.

Terry, R. D. (1971). Neuronal fibrous protein in human pathology. Journal of Neuropathology and Experimental Neurology, 30, 8-19.

Toghill, P. J., Williams, R., Stephens, J. D., and Carroll, J. D. (1969). Acute hepatic necrosis following an overdose of paracetamol. Gastroenterology, 56, 773-776.

Walberg, F. (1964). The early changes in degenerating boutons and the problem of argyrophilia. Light and electron microscopic observations. Journal of Comparative Neuro$\log y, 122,113-137$.

Walberg, F., and Mugnaini, E. (1969). Distinction of degenerating fibres and boutons of cerebellar and peripheral origin in the Deiters' nucleus of the same animal. Brain Research, 14, 67-75.

Weiss, P. A., and Mayr, R. (1971). Organelles in neuroplasmic ('axonal') flow: neurofilaments. Proceedings of the National Academy of Sciences of the United States of America, 68, 846-850.

Wiśniewski, H., Terry, R. D., and Hirano, A. (1970). Neurofibrillary pathology. Journal of Neuropathology and Experimental Neurology, 29, 163-175. 